\title{
Hybrid numerical analysis of a high-speed non-volatile suspended gate silicon nanodot memory (SGSNM)
}

\author{
Mario A. García-Ramírez • Yoshishige Tsuchiya • \\ Hiroshi Mizuta
}

Published online: 5 May 2011

(C) Springer Science+Business Media LLC 2011

\begin{abstract}
We present a hybrid numerical analysis of a high-speed and non-volatile suspended gate silicon nanodot memory (SGSNM) which co-integrates a nano-electromechanical (NEM) control gate with a MOSFET as a readout element and silicon nanodots as a floating gate. A hybrid NEM-MOS circuit simulation is developed by taking account of the pull-in/pull-out operation of the suspended gate and electron tunnelling processes through the tunnel oxide layer as behavioural models. The signals for programming, erasing and reading are successfully achieved at circuit level simulation. The programming and erasing times are found as short as $2.5 \mathrm{nsec}$ for a SGSNM with a 1- $\mu \mathrm{m}$-long suspended gate, which is a summation of the mechanical pull-in/pullout times and the tunnel charging/discharging times.
\end{abstract}

Keywords Non-volatile memory $\cdot$ Nano electromechanical Systems (NEMS) · Suspended gate structure · Hybrid circuit simulation $\cdot$ Electron tunelling process

\section{Introduction}

The non-volatile memory market has been driven by Flash memory in the last three decades. Flash memory is currently used for a variety of devices and systems from pen drives and mobile phones to large systems in cars or planes. However, the conventional floating-gate (FG) based technology is facing a serious scalability issue as reported in the latest ITRS2009 [1]. The main drawback of downscaling flash

M.A. García-Ramírez ( $₫)$ · Y. Tsuchiya · H. Mizuta

Nano Group, School of Electronics and Computer Science, University of Southampton, SO17 1BJ Southampton, UK

e-mail: magr07r@ecs.soton.ac.uk memory is the increased leakage current via the gate oxide layer. The leakage issue is caused by the defect states formed in the tunnel oxide during the programming/erasing $(\mathrm{P} / \mathrm{E})$ operation based on the channel hot electron injection or Fowler-Nordheim tunnelling processes [2-4]. In order to avoid this leakage issue, an alternative FG structure which adopts silicon nanodots (SiNDs) rather than a solid polysilicon was proposed $[5,6]$. The SiNDs are single crystals surrounded by a thin $\mathrm{SiO}_{2}$ isolation layer [7, 8]. In case of a leakage path formation within the tunnel oxide layer, only the electrons stored adjacent to the path might leak and as consequence the total loss of charge stored in the FG is expected to decrease. However, this technique cannot solve the leakage current issue fully and alternative approaches are sought in order to achieve a superior non volatility.

One such new approach is to integrate a movable nanoelectromechanical (NEM) structure into memory devices [9-11]. The first NEM non-volatile memory was a selfbuckled FG memory which adopts a mechanically-bistable FG [11]. The self-buckled FG was made with a SiNDs monolayer sandwiched between $\mathrm{SiO}_{2}$ layers and doubly clamped in a cavity integrated on the readout MOSFET. The SiNDs embedded in the FG were initially charged with electron injection from top control gate before removing an upper sacrificial $\alpha$-Si layer [12]. The self buckled FG flip-flops between its mechanically-bistable states by applying a voltage to the top control gate, which changes the MOSFET surface potential, resulting in the shift of the threshold voltage. As the $\mathrm{P} / \mathrm{E}$ processes do not involve the charge tunnelling via the gate oxide layer, there is no degradation of this layer, and therefore no leakage current is anticipated. However, this NEM memory has inherently an unfavourable tradeoff between the P/E voltage and readout current ON/OFF ratio and a recent study indicates that the scalability deteriorates when the beam length is scaled down to less than $100 \mathrm{~nm}$ [12]. In 


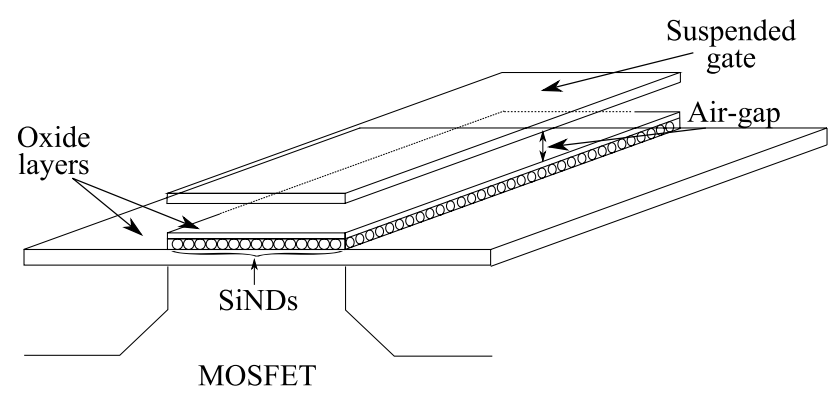

Fig. 1 Schematic diagram of the suspended gate silicon nanodot memory (SGSNM) cell

order to surpass this issue, we propose a new suspended gate silicon nanodot memory (SGSNM) that features a movable top gate suspended over a fixed FG layer [13].

The SGSNM uses a MOSFET as readout element, a silicon nanodots monolayer as FG and a movable suspended control gate (SG) which is isolated from the FG by an oxide layer and by an air gap as shown in Fig. 1.

This structure performs the programming and erasing processes through dynamic pull-in/pull-out motions of the SG. By applying a negative bias voltage on the SG electrode, the SG bends downwards and contacts the gate oxide layer allowing electrons to tunnel through it into the FG. When the applied voltage is reduced below the pull-in voltage, the SG remains attached to the tunnel oxide layer. This phenomenon occurs because the stiction force such as the van der Waals force is in equilibrium with the SG restoring force. Further reduction in the applied voltage produces an unbalance between those forces allowing the SG to return to its isolated flat position (pull-out effect). After the $\mathrm{SG}$ is disengaged, the readout element is activated to read the memory node state. When negative charge is read in the memory node, it is indicative that the memory has been programmed. Therefore, no current conduction is displayed by the readout device (programmed). On the other hand, when a positive bias voltage is applied on the SG, a similar process as described above occurs but with the main difference that the electrons are removed from the FG. Considering that the pull-out effect occurs at a fixed point for $\mathrm{P} / \mathrm{E}$ processes, the memory node is positively charged. When the readout element reads that the FG is positively charged, it is indicative that the SGSNM has been erased and as consequence a current pulse is displayed by the MOSFET [14]. In the SGSNM the voltage at which the P/E processes occur can be designed via a few parameters such as material, length and air gap thicknesses. In addition, the amount of charge stored within the FG is designed via the number of SiNDs and the isolation material in which the SiNDs are surrounded. The capability to design separately the P/E voltage and the readout current ON/OFF ratio allows the SGSNM to overcome the scalability issue and the ON/OFF tradeoff present on the

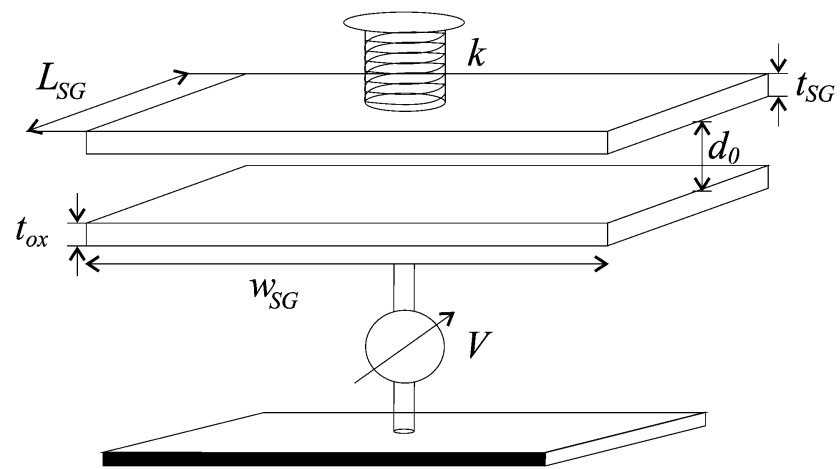

Fig. 2 Double-plate capacitor model used to obtain the pull-in equation

self-buckled NEM device. Moreover, thanks to the presence of the $\mathrm{SG}$, it is possible to have virtually no leakage current and reach high $\mathrm{P} / \mathrm{E}$ speed processes. In this paper, we analyse the SGSNM structure. This analysis is divided into three parts. The first part focuses on the electro-mechanical analysis of the SG (pull-in) analytically and numerically by using finite element method. The second part studies the quantummechanical tunnelling process that allows the electrons to tunnel through the gate oxide layer as function of the voltage applied on the SG. Finally, the curves obtained from above analysis are implemented as behavioural models within an SGSNM equivalent circuit in an analog circuit simulation.

\section{Numerical analysis of the suspended gate}

One of the criteria of the SGSNM is low-voltage P/E operation. The $\mathrm{P} / \mathrm{E}$ voltage is defined as the pull-in voltage that strongly depends on structural parameters as well as in some physical properties [9, 15-17]. In this structure, the suspended control gate is analysed through a parallel-plate capacitor model that considers the Poisson's ratio $(n)$, Young modulus $(E)$ and air gap $\left(d_{0}\right)$ as key parameters. Those parameters define at which voltage the pull-in effect will occur.

Figure 2 shows the doubly-plate capacitor model where $L_{S G}$ and $w_{S G}$ are the length and width of the beam, $d_{0}$ is the initial air gap, $t_{S G}$ is the beam thickness, $V$ is the applied voltage and $k$ is the spring constant. The spring constant depends on the structural shape such as membrane, cantilever or beam [17-19]. For a doubly clamped beam, the required spring constant is defined as

$k=\frac{16 E w_{S G} t_{S G}^{3}}{L_{S G}^{3}}$

According to the parallel-plate capacitor model, the pullin voltage is defined as 
Table 1 Parameters used to model the doubly-clamped beam structure

\begin{tabular}{lcllc}
\hline $\begin{array}{l}\mathrm{SG} \\
\text { material }\end{array}$ & $\begin{array}{l}E \\
(\mathrm{GPa})\end{array}$ & $\begin{array}{l}\text { Dielectric } \\
\text { permittivity }\end{array}$ & $\begin{array}{l}\text { Dimensions } \\
\left(L_{S G} \times w_{S G}\right)(\mathrm{nm})\end{array}$ & $\begin{array}{l}\text { Thickness } \\
(\mathrm{nm})\end{array}$ \\
\hline $\mathrm{Al}$ & 77 & & $1000 \times 250$ & 30 \\
$\mathrm{Cu}$ & 128 & & $1000 \times 250$ & 30 \\
$\mathrm{Poly}-\mathrm{Si}$ & 160 & & $1000 \times 250$ & 30 \\
$\mathrm{Si}$ & 169 & & $1000 \times 250$ & 100 \\
$\mathrm{SiO}_{2}$ & 73 & 3.8 & $1000 \times 250$ & 10 \\
$\mathrm{Si}_{3} \mathrm{~N}_{4}$ & 222 & 7 & $1000 \times 250$ & 10 \\
Air gap & & 1.0059 & & 40 \\
\hline
\end{tabular}

$V_{\text {pull-in }}=8 \sqrt{\frac{2 E t_{S G}^{3} d_{0}^{3}}{27 \varepsilon_{0} L_{S G}^{4}}}$

Pull-out voltage is defined as

$V_{\text {pull-out }}=\sqrt{\frac{2 k t_{o x}^{2}}{\varepsilon_{0} \varepsilon_{o x} A}\left(d_{0}-t_{o x}\right)}$

where $A$ is defined as the plate area, $t_{o x}$ as the oxide thickness, $\varepsilon_{0}$ is the free space permittivity and $\varepsilon_{o x}$ is defined as the oxide permittivity.

Parallel-plate capacitor model considers the electrostatic and electromechanical forces as the unique forces acting in the pull-in/pull-out effects but other forces based on different nature as the quantum mechanical processes $[15,17]$. To complement the pull-out analysis an extra quantummechanical term as the van der Waals force that describes the interaction force at small gaps is manually added in the pull-out voltage equation [20]. Therefore, the pull-out equation is re-defined as

$V_{\text {pull-out }}=\sqrt{\frac{2 k t_{o x}^{2}}{\varepsilon_{0} \varepsilon_{o x} A}\left(d_{0}-t_{o x}\right)-\frac{A_{h}}{3 \pi \varepsilon_{o x} t_{o x}}}$

where $A_{h}$ is defined as the Hammaker constant.

Equations (2) and (4) describe the voltage at which the pull-in and pull-out effects occur without considering stress, tension or compression that plays a significant role in the doubly-clamped beam structure. In order to include those electro-mechanical properties, the parallel-plate capacitor structure is analysed by using 3-dimensional (3D) finite element method (FEM) [16, 17]. Table 1 summarises the set of parameters and materials used in this task. Figure 3 shows the parallel-plate capacitor model implemented and analysed in a 3D FEM software [17].

Figure 4 shows the group of pull-in trajectories obtained as a result of the 3D FEM electro-mechanical analyses.
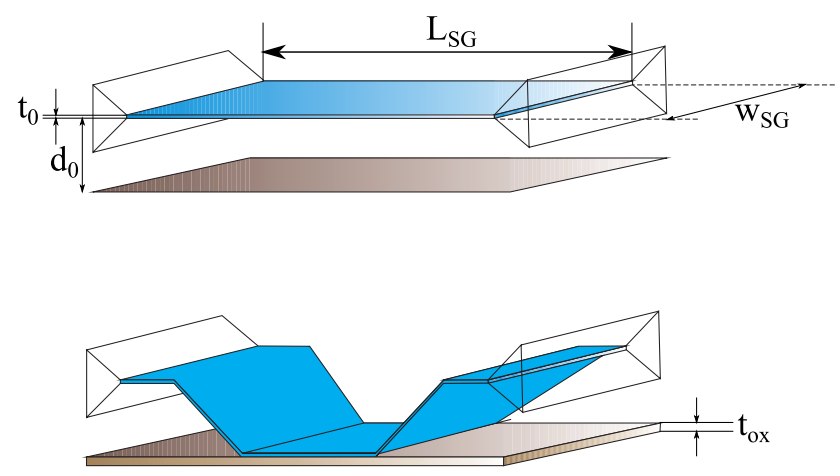

Fig. 3 Schematic diagram of the doubly-clamped beam structure, (a) for pull-in effect and (b) for pull-out effect

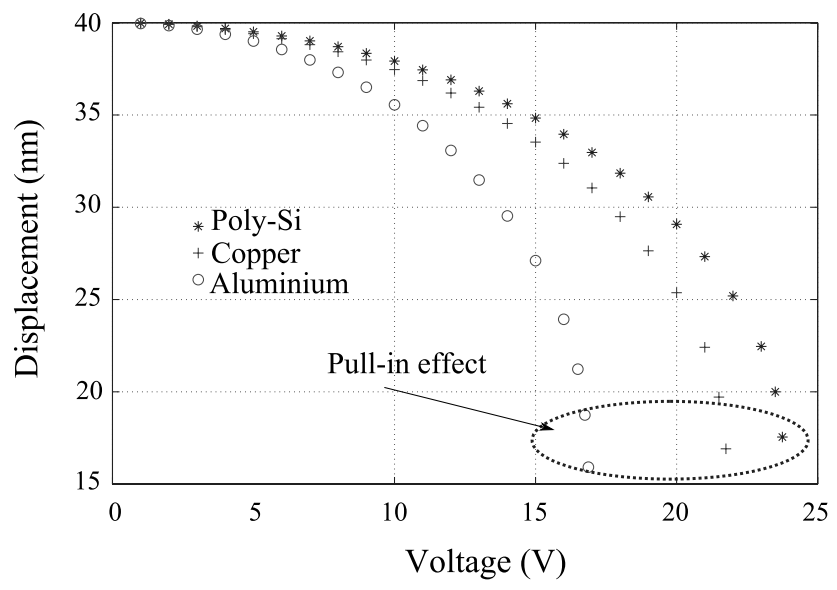

Fig. 4 Pull-in trajectories analysed for a set of doubly-clamped beams made of $\mathrm{Al}, \mathrm{Cu}$ and Poly-Si

From those pull-in trajectories, the aluminium-beam based is chosen as SG material because it shows low pull-in voltage as well as a sharp transition [15].

To obtain the pull-out voltage the SG is coupled to a tunnel oxide layer as shown in Fig. 3b. In this 3D FEM analysis the Al-SG is coupled to $\mathrm{SiO}_{2}$ and $\mathrm{Si}_{3} \mathrm{~N}_{4}$ tunnel oxide layers, respectively and a bias voltage is swept on the $\mathrm{SG}$ electrode from 0 to $25 \mathrm{~V}$ and vice versa. As a result, the pull-in and pull-out voltage curves are obtained and merged generating a displacement-voltage $(D-V)$ hysteresis cycle as depicted in Fig. 5.

When both hysteresis curves are overlapped as depicted in Fig. 5, it shows that pull-in voltage for both analyses converges at the same point despite the tunnel oxide permittivity variation $\left(\mathrm{SiO}_{2}=3.8, \mathrm{Si}_{3} \mathrm{~N}_{4}=7.1\right)$ [21]. In contrast, the small variation in permittivity creates a large change in the pull-out voltage for both structures generating a change of $2 \mathrm{~V}$ in the stiction window. As a result of this analysis the pull-out voltage obtained with $\mathrm{SiO}_{2}$ shows the shortest stiction window. Thus, it is used as the tunnel oxide layer.

Once obtained, the $D-V$ hysteresis cycle shows a comparatively high programming voltage within the present 


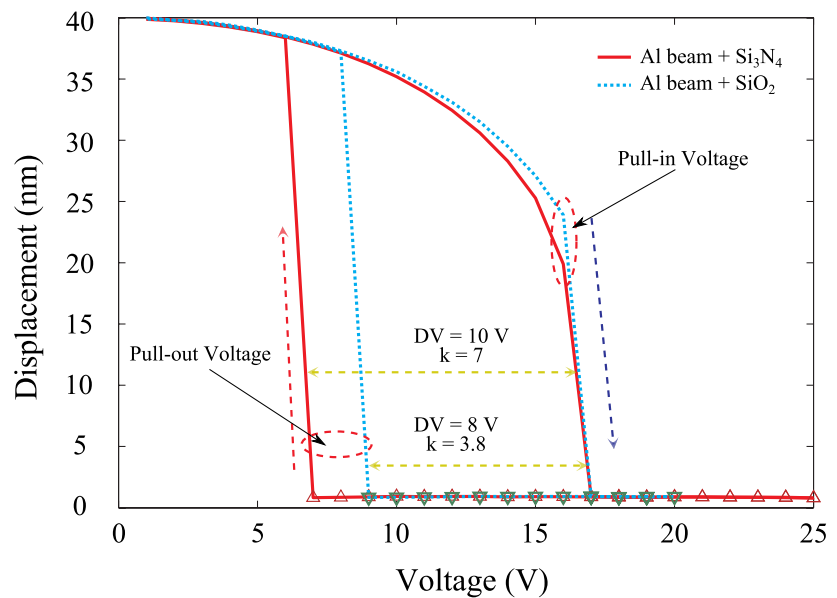

Fig. 5 Hysteresis curves obtained for a doubly-clamped beam structure in which $\mathrm{Al}$ beams were coupled to $\mathrm{SiO}_{2}$ and $\mathrm{Si}_{3} \mathrm{~N}_{4}$ layers

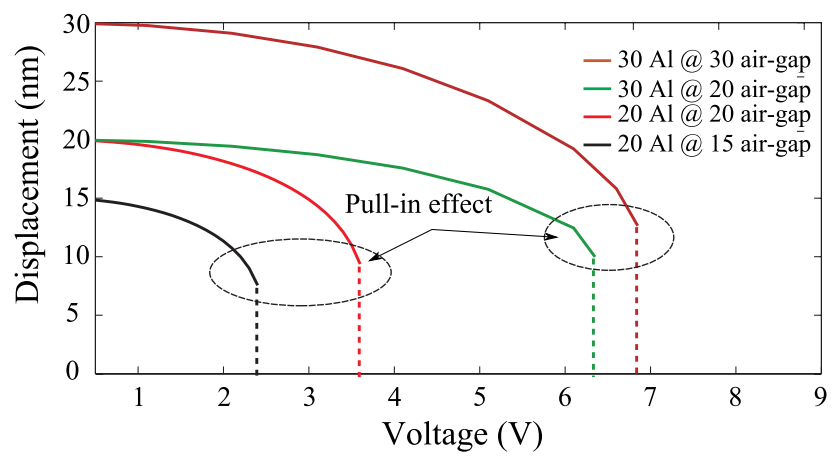

Fig. 6 Pull-in trajectories obtained for the set of parameters specified in Table 2 in which those voltages are below $10 \mathrm{~V}$

Table 2 Structural parameters for a low-voltage programming sequence

\begin{tabular}{llll}
\hline $\begin{array}{l}\text { Material } \\
\text { SG }\end{array}$ & $\begin{array}{l}\text { SG } \\
d_{0}(\mathrm{~nm})\end{array}$ & $\begin{array}{l}\text { Air gap } \\
(\mathrm{nm})\end{array}$ & $\begin{array}{l}\text { Dimensions } \\
L_{S G}(\mu \mathrm{m}) \times w_{S G}(\mathrm{~nm})\end{array}$ \\
\hline $\mathrm{Al}$ & 30 & 30 & $1 \times 300$ \\
$\mathrm{Al}$ & 30 & 20 & $1 \times 300$ \\
$\mathrm{Al}$ & 20 & 20 & $1 \times 300$ \\
$\mathrm{Al}$ & 20 & 15 & $1 \times 300$ \\
\hline
\end{tabular}

non-volatile memory market requirements [1]. Therefore, in order to be competitive within the non-volatile memory market, the programming voltage for the SGSNM is settled to be lower than $10 \mathrm{~V}$. In order to fulfill those requirements Table 2 summarises a set of structural parameters that are analysed in order to get the above goal.

Figure 6 shows the pull-in curves obtained, those parameters fulfill the SGSNM programming voltage requirements. From those pull-in curves, the $30 \mathrm{~nm} \mathrm{SG}$ and $20 \mathrm{~nm}$ air gap

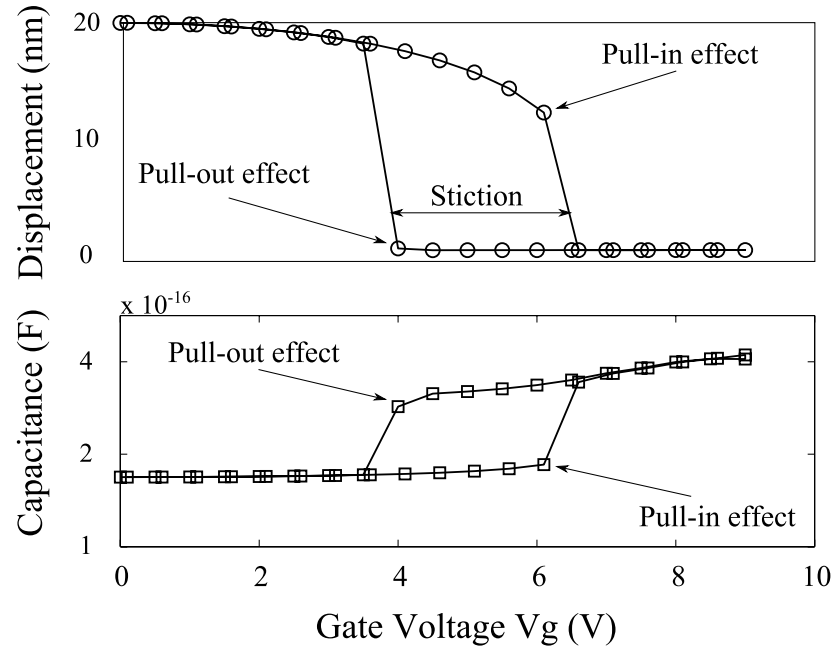

Fig. 7 Hysteresis cycles for a $30 \mathrm{~nm}$ Al beam at $20 \mathrm{~nm}$ air gap. (a) Displacement-voltage, showing the pull-in/pull-out voltages with stiction; (b) capacitance-voltage behaviour

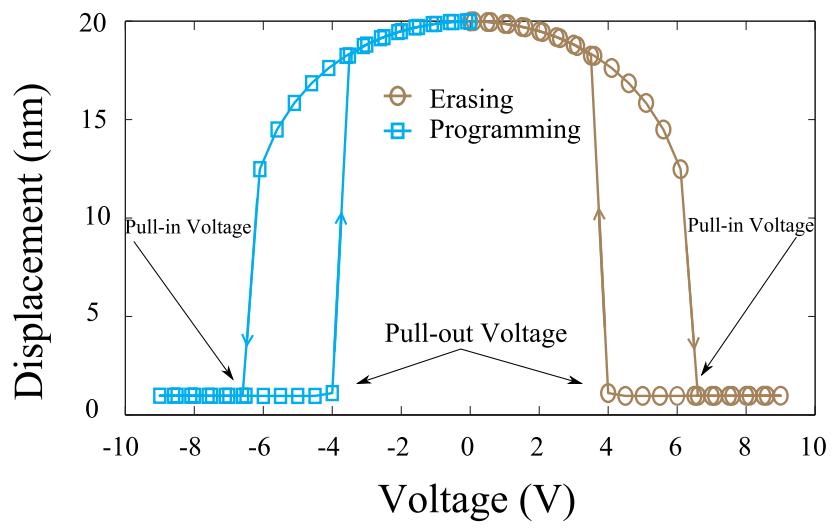

Fig. 8 Programming and erasing hysteresis curves according to the SGSNM cell

were chosen as structural parameters because of the pull-in voltage and the stiffness provided by the thickness of the SG.

The pull-in analysis is complemented by coupling the doubly-clamped beam with a $\mathrm{SiO}_{2}$ layer. As the pull-in voltage is lower than $10 \mathrm{~V}$, a bias voltage was swept from 0 to $10 \mathrm{~V}$ and vice versa for the pull-in/pull-out analysis. As a result, the $D-V$ and the capacitance-voltage $(C-V)$ hysteresis curves were obtained as depicted in Fig. 7.

In the SGSNM the programming and erasing processes strongly depend on the bias voltage applied on the SG electrode. When a negative voltage is applied on the SG electrode, the SGSNM is going to be programmed. In contrast, when a positive voltage is applied on the SG electrode, the memory node is erased as depicted in Fig. 8.

Programming and erasing processes are implemented as electrical behavioural models rather than mechanical within analog models. Therefore, the $C-V$ hysteresis cycle is trans- 


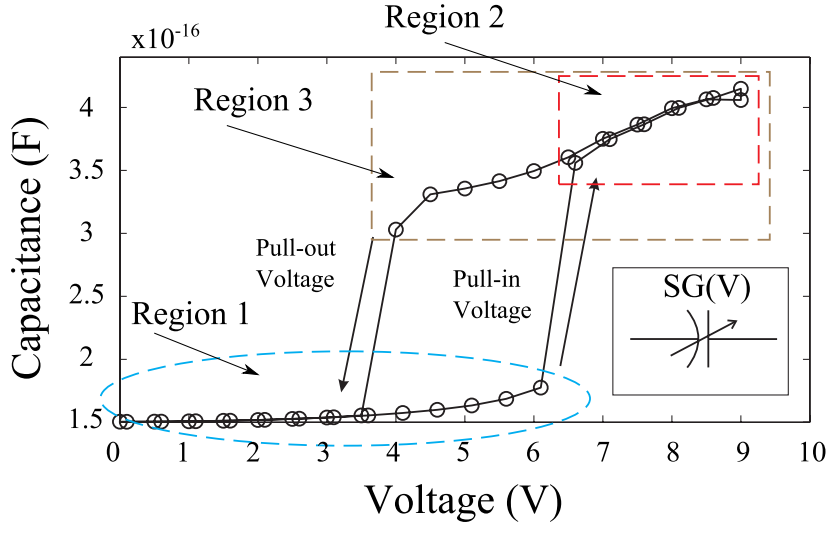

Fig. $9 C-V$ hysteresis curve divided in 3 regions that represents the pull-in, stiction and pull-out voltage dependent functions

formed into three analog voltage dependent functions as depicted in Fig. 9 (Appendix A). Discrete curves are transformed into analog functions. This transformation is implemented through algebraic algorithms rather than complex and heavy, computationally speaking, mathematical functions. The implementation of those voltage dependent functions into the numerical analysis is performed by using a hardware description language [22, 23]. The language that we used to implement those functions is Verilog-AMS $[23,24]$. Equivalent circuit model for the voltage dependent functions is depicted inside Fig. 9.

Once the pull-in/pull-out $C-V$ characteristic curves have been obtained, it is required to know the time at which the Al 1- $\mu \mathrm{m}$-long SG collapses on the tunnel oxide layer. Hence, in order to find the time at which the pull-in effect occurs, a 2D-SG FEM transient analysis is performed [16]. This numerical analysis considers the electrostatic as well as the Casimir force interaction between the SG and the tunnel oxide layer.

From this analysis it was found that for a 1- $\mu \mathrm{m}$-long beam the time at which the pull-in effect occurs for the P/E processes is $0.8 \mathrm{nsec}$ as depicted in Fig. 10. In addition to the mechanical analysis, it is pointed out that the SGSNM cell might present an endurance issue related to clamp fracture due to hinge fracture. This issue is produced by the electromechanical operation of the doubly-clamped beam structure. We have not studied it yet owing to difficulty of simulating the endurance problem. Nevertheless, it was demonstrated qualitatively that for a similar doubly-clamped structure and by using a stiffest material (poly-Si), a sample was tested over $10^{5}$ cycles without show fatigue or mechanical degradations [25]. Based on those results and taking into account that the material used within the SGSNM cell is aluminium (lower stiffness), we are confident that the SGSNM cell can meet the $10^{6}$ cycles similar to Flash memory or higher. Finally, the energy required to move the SG is a

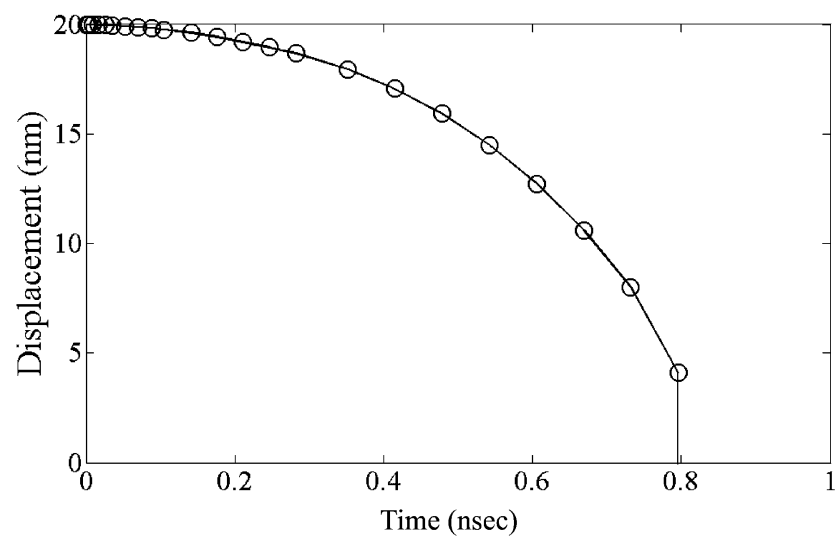

Fig. 10 Characteristic curve of a transient analysis performed to calculate the mechanical switching time (pull-in effect) of the SG

combination between several kinds of energies such as elastic energy $E_{m}$, kinetic energy $E_{k}$, damping loss energy $E_{d}$, electrostatic energy $E_{e}$ and charging loss energy $E_{R}$ [26]. A detailed analysis for energy and power consumption is being processed which results will be included in further publications.

\section{Quantum-mechanical tunnelling process: current density analysis}

The tunnelling process for the programming and erasing processes in the SGSNM is performed through a thin tunnel oxide layer $\left(\mathrm{SiO}_{2}\right)$. This oxide layer is modelled as a quantum mechanical squared-profile potential barrier (Fig. 11) [27].

When a negative bias voltage is applied on the SG, it bends and collapses on the tunnel oxide layer allowing the quantum mechanical Fowler-Nordheim tunnelling process to occur (Fig. 11b). Whilst the applied voltage is reduced, the tunnel barrier shape recovers its original form. Once recovered, the electrons remain trapped within the FG as depicted in Fig. 11c. In contrast, when a positive voltage is applied on the SG, the tunnel barrier bends downwards until it becomes triangular allowing the electrons to tunnel from the FG into the SG as depicted in Fig. 11e. When the applied voltage is reduced, the tunnel oxide barrier recovers its original form letting the FG positively charged (Fig. 11f).

The quantum-mechanical tunnelling process is analysed numerically by co-solving self-consistently the Poisson's and 1D-Schrödinger equations [27]. Those equations are solved by using the algorithm that makes use of the TsuEsaki formula and the Transfer Matrix Method [2, 27]. The combination of those tools calculates the current-density $(J)$ through an arbitrary-shaped one-dimensional tunnelling barrier [27]. 
Fig. 11 Programming and erasing sequence according to the SG and tunnelling processes. (a) SG without bias,

(b) a negative voltage is applied on the SG and the electrons tunnelling is allowed because of the triangular shape of the tunnel barrier. (c) By reducing the applied voltage the SG returns as well as the tunnel barrier to its initial position. (d) Considering the SGSNM cell programmed, a positive voltage is applied on the SG (d) and the electrons are removed from the FG (e), by reducing the applied voltage both the SG and the tunnel barrier return to their initial position (f) a)
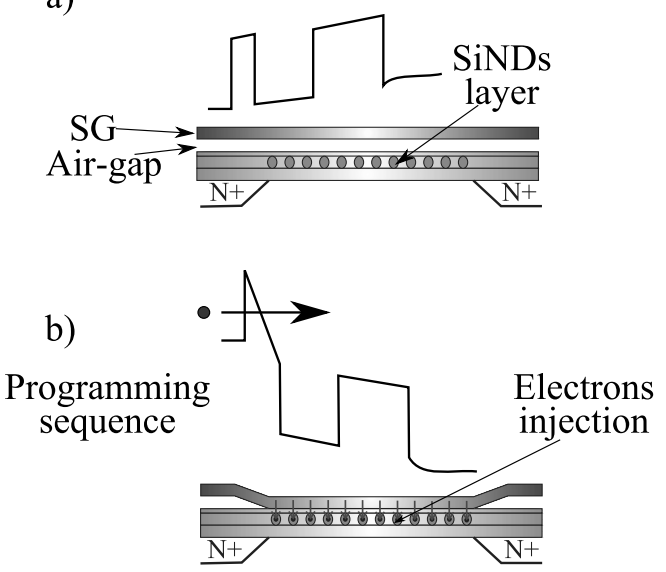

c)

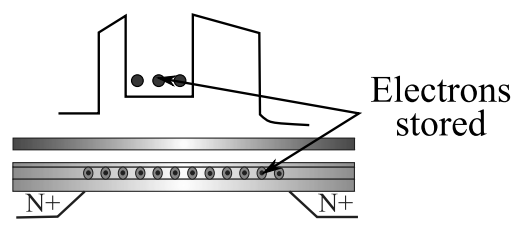

d)

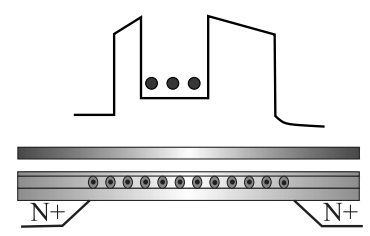

e)

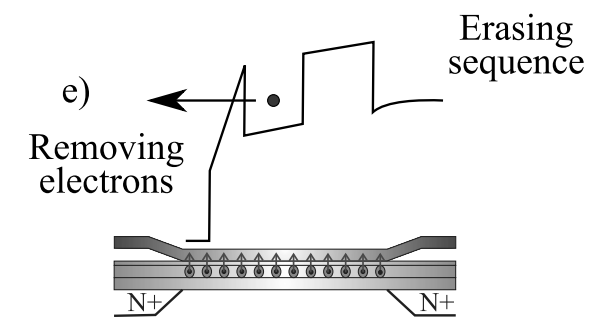

f)

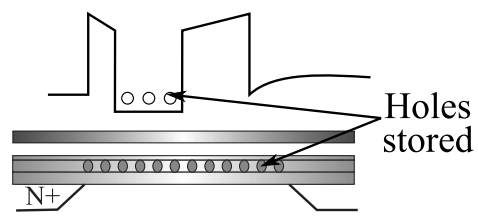

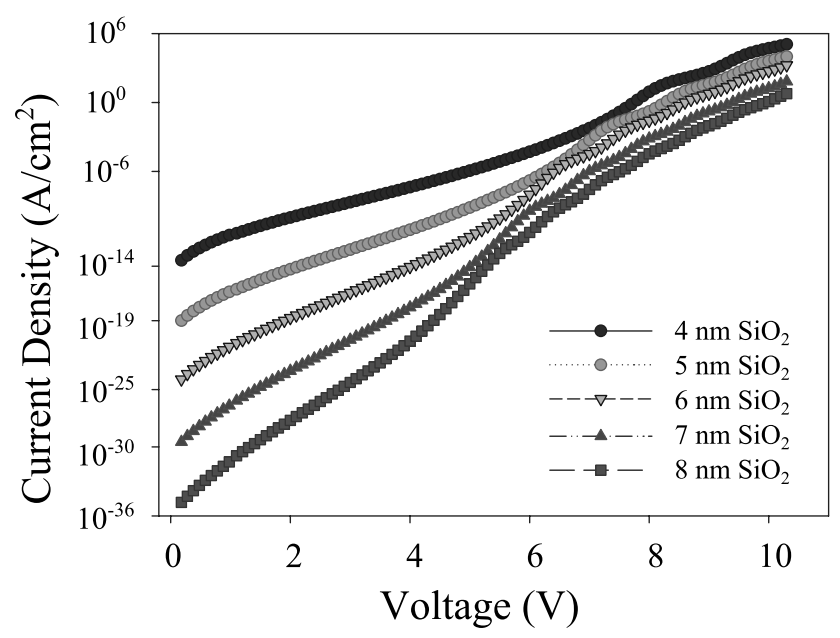

Fig. 12 Characteristic current density-voltage curves for a set of $\mathrm{SiO}_{2}$ thicknesses

Those equations are implemented in a homemade FEM algorithm in which a set of $\mathrm{SiO}_{2}$ thicknesses are analysed. This set of thicknesses goes from 4 to $8 \mathrm{~nm}$ and the span of applied voltage is similar as for the SG analysis $(0$ to $10 \mathrm{~V})$. Once performed, the FEM analysis shows the current-density-voltage $(J)$ characteristic curve for each thickness as depicted in Fig. 12.

Figure 12 shows a fairly large tunnel resistance at low bias generates a negligible current density through the tunnel barrier. It shows a $J-V$ curve variation between each thickness as much as 5 orders of magnitude. Once the applied voltage reaches the pull-in voltage, the tunnel resistance decreases several magnitude orders as Fowler-

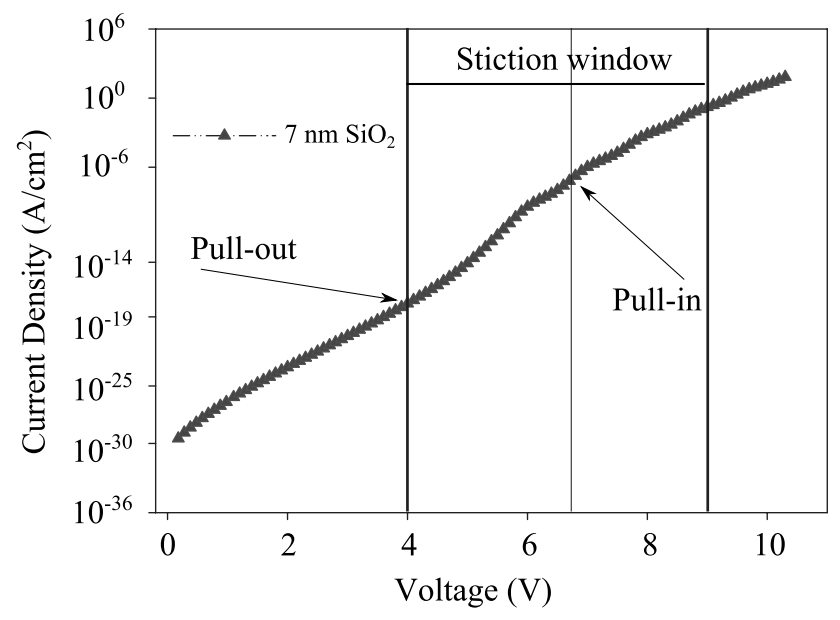

Fig. 13 Characteristic current density-voltage curve for a $7 \mathrm{~nm} \mathrm{SiO} 2$ layer

Nordheim tunnelling process states. Hence, $J$ increases several magnitude orders. At high bias voltage ( $>15 \mathrm{~V})$, tunnel resistance is quite low so that $J$ reaches a saturation point.

From that $J-V$ set of curves, the $7 \mathrm{~nm} \mathrm{SiO}_{2}$ layer is arbitrarily selected as the tunnel oxide layer of the SGSNM. Considering that the tunnelling process is only present after the pull-in process occurs until the pull-out takes place (stiction window), the $J-V$ curve is divided into two sections as depicted in Fig. 13.

The sections in which the $J-V$ curve has been divided are transformed from discreet voltage functions into analog voltage dependent functions by using Verilog-AMS as 
shown elsewhere (Appendix B). The equivalent circuit that represents the tunnelling process and the floating gate are sketched as a variable tunnel resistor in parallel with a tunnel capacitor (Fig. 14). In this model, the tunnel capacitor sketches the memory node that is considered as a SiNDs monolayer. The SiNDs diameter is controlled to be

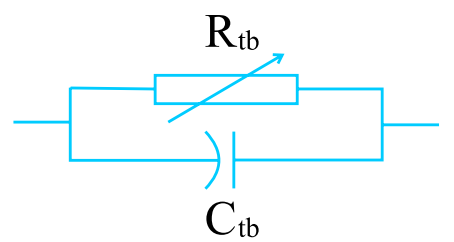

Fig. 14 Tunnelling barrier equivalent circuit. Where $\mathrm{C}_{t b}$ is the tunnelling capacitance and $\mathrm{R}_{t b}$ is the tunnelling voltage dependent resistance

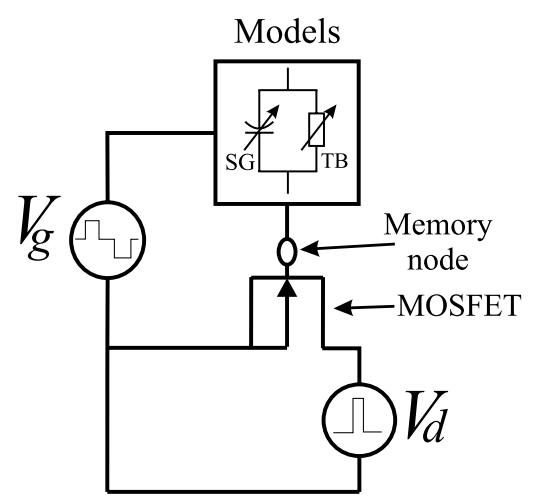

Fig. 15 Schematic diagram of the equivalent circuit of the suspended gate silicon nanodot memory (SGSNM) cell
$10 \pm 1 \mathrm{~nm}$ and the SiNDs distribution over the sample is performed through the Langmuir-Blodgett technique having a density up to $7 \times 10^{11} \mathrm{~cm}^{-2}$ [28]. By oxidising the sample a thick isolation layer between them is formed. This separation is large enough to avoid any lateral charge migration, this is why it was not included in the analysis [29].

It should also be noted that we simply ignored a temporal variation of a triangular potential barrier profile and therefore a decrease in the Fowler-Nordheim tunnelling current density during the $\mathrm{P} / \mathrm{E}$ processes. This may result in an overestimation of the speed of the P/E processes whose quantitative evaluation is left for our future work.

\section{Numerical implementation of the SGSNM}

The SGSNM is simulated numerically by using an equivalent circuit model depicted in Fig. 15 which considers the implementation of the SG and tunnel oxide layer as behavioural models, a MOSFET as readout element, a pulse source is used as drain signal and a bi-stable piece wise linear source as control gate [31].

From this simulation a chart of signals that describes the memory behaviour is obtained as depicted in Fig. 16.

The chart of signals obtained from the circuit simulation of the SGSNM cell displays the behaviour of each node at programming $(\mathrm{P})$, reading $(\mathrm{R})$ and erasing operations. The follow process program-read-erase-read start when a negative voltage is applied on the control gate as depicted in Fig. $16 \mathrm{a}(\mathrm{P})$. At this point, the memory node shows how the electrons are injected into the FG (Fig. 16c(P)) by action
Fig. 16 Chart signals of the main nodes for the equivalent circuit of the SGSNM cell. (a) SG voltage, (b) drain MOSFET voltage, (c) memory node and $(\mathbf{d})$ readout current MOSFET

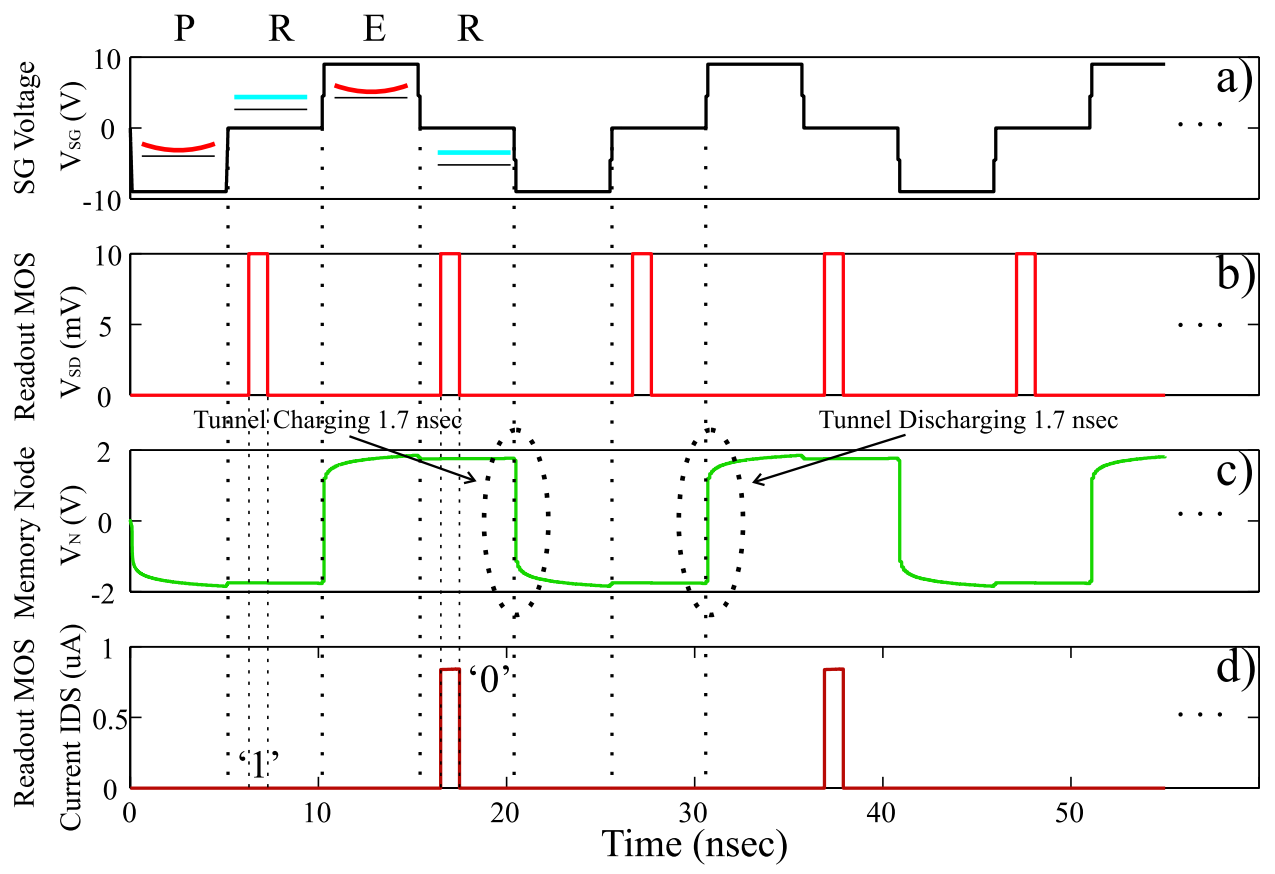




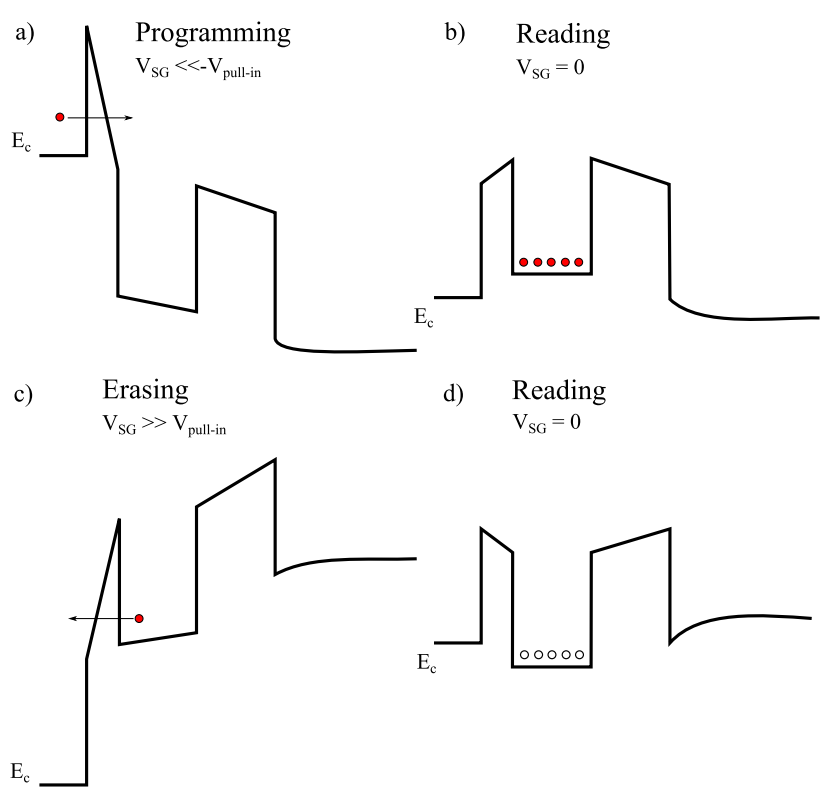

Fig. 17 Band diagram structure for the SGSNM cell. (a) Illustration of programming process: electron tunnelling process from the SG into the silicon nanodots. (b) Negatively-charge stored floating gate, (c) illustration of the erasing process: electron tunnelling process form the FG into the SG, and (d) positively-charge stored floating gate

of the Fowler-Nordheim tunnelling mechanism described as a band diagram by Fig. 17a. When the programming sequence concludes, the reading process takes place. In the floating gate, it is possible to see a negative voltage indicating that electrons have been trapped within the SiNDs and the FG is negatively charged (Fig. 16c(R) and 17b). In here, drain source is biased and the readout element displays no-current pulse indicating that the SGSNM is programmed (Fig. 16d(R)). Continuing with the initial sequence, to erase the memory node a positive voltage is applied on the control gate. The floating gate shows how the electrons are removed from the FG into the SG (Fig. 16c(E) and 17c). Once the erasing process concludes, it shows the floating gate positively charged (Fig. 16c(R) and 17d). When readout element is activated, it displays a current pulse indicating that the memory node is erased and the cycle starts over again as depicted in Fig. 16.

The programming and erasing times can not be separated due to both times are linked to the control voltage and to the electro-mechanical movement of the SG. The combination of the mechanical switching time obtained from the transient FEM analysis ( $0.8 \mathrm{nsec})$ with the charging/discharging time obtained from the circuital simulation $(1.7 \mathrm{nsec})$ shows that the time required for both programming and erasing operations in the SGSNM cell is symmetrical $(2.5 \mathrm{nsec})$. For the reading time, it is expected, in principle to be $80 \mathrm{nsec}$ similar as for the conventional SiNDs floating gate memories and comparable to conventional flash memories [30]. The

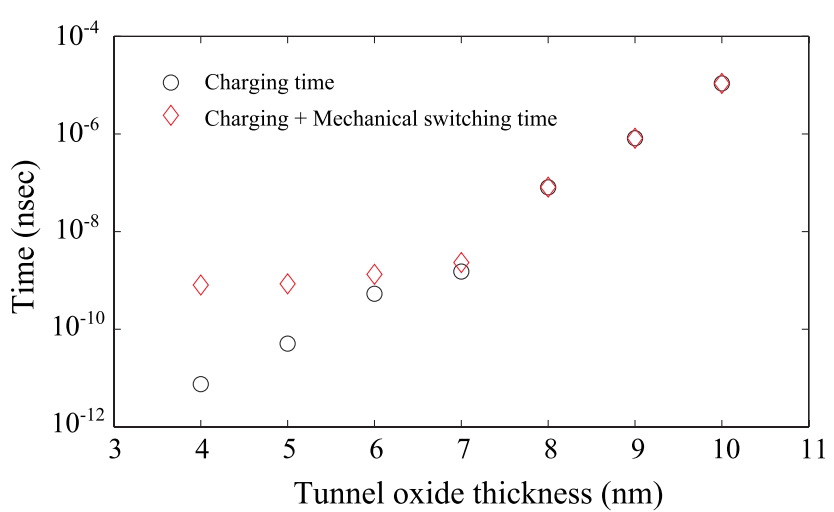

Fig. 18 Switching time due to the charging and charging + mechanical switching time of the SGSNM cell

programming and erasing time are strongly related to few structural parameters such as SG material and thickness, airgap and tunnel oxide permittivity. Considering those times can be improved by modifying some parameters, an extra analysis is conducted in this direction. In here, few parameters within the SG structure such as area, thickness and SG/air-gap ratio are kept fixed whilst tunnel oxide layer is varied. As shown elsewhere (Fig. 12), the current density increases as tunnel oxide thickness decreases. According to this premise a set of tunnel oxide layers is analysed within a circuit simulation. The analysis considers $\mathrm{SiO}_{2}$ as tunnel oxide layer and the layers under test goes from 4 to $10 \mathrm{~nm}$ thick. Figure 18 shows the P/E times obtained as function of the tunnel oxide layer for the charging/discharging times and as a combination with the mechanical switching time.

This analysis shows that by maintaining few parameters fixed, the charging/discharging time in the memory node is improved by few tens of nanoseconds whilst the tunnel oxide layer becomes thinner $(4 \mathrm{~nm})$. Based on the P/E time improvement and by scaling-down properly the SG structural parameters, it is viable to reach even higher $\mathrm{P} / \mathrm{E}$ times.

The scaling-down process of the memory cell is linked to the relationships between the SG structural parameters such as beam length, thickness and air-gap with the shortrange forces (Casimir and van der Waals forces). This imply that the shorter the air-gap, the stronger the short-range forces becomes. Those forces are expected to have strong influence on the SG behaviour when the air-gap reaches a separation of $1 \mathrm{~nm}$ [32]. Therefore, the SG is designed considering those forces in order to get a competitive trade-off between beam stiffness and operation voltage

\section{Summary}

We presented a hybrid numerical analysis of the Suspended Gate Silicon Nanodot Memory (SGSNM). The signals for 
programming, reading and erasing were achieved at circuit level simulation. The programming and erasing times were found as short as $2.5 \mathrm{nsec}$ for a $1-\mu \mathrm{m}$-long suspended gate which is a summation of the mechanical pull-in/pullout times and the tunnel charging/discharging times for a $7 \mathrm{~nm} \mathrm{SiO} 2$ tunnel oxide layer. Those superior characteristics along with the manufacturability of the SGSNM within conventional silicon fabrication processes without the introduction of any "exotic" material makes this memory device a serious candidate for non-volatile random access memory applications.

Acknowledgements The authors wish to thank Prof. S. Oda and his laboratory members of Tokyo Institute of Technology for valuable discussions. One of the authors (M.A. García-Ramírez) would like to thank the National Council of Science and Technology (CONACyT), México.

\section{Appendix A: Suspended gate implementation}

The implementation of the $C-V$ hysteresis curve as behavioural models implies the transformation of the threediscrete regions into several analog voltage dependent functions (see Fig. 19). The approximation of each one of those regions was made by using a polynomial algorithm rather than implementing a complex and heavy one (computationally speaking). The algorithm that was used approximates each curve by using a third degree polynomial which is easy to implement and is defined as

$$
F(V)=A+B V+C V^{2}+D V^{3}
$$

The coefficients for each region are shown in Table 3.

Above equation describes the erasing process. For the full $\mathrm{P} / \mathrm{E}$ processes, the equations are mirrored and implemented as depicted in Fig. 20.

\section{Appendix B: Tunnel barrier implementation}

The tunnelling process occurs while the SG remains in contact with the tunnel oxide layer. This tunnelling process $(J-V$ curve) was transformed from discreet into analog voltage dependent functions (Fig. 21). The model that

Table 3 Structural parameters for a low-voltage programming sequence

\begin{tabular}{lccc}
\hline Variable & $\begin{array}{c}\text { Region 1 } \\
\times 10^{-16}\end{array}$ & $\begin{array}{c}\text { Region 2 } \\
\times 10^{-16}\end{array}$ & $\begin{array}{c}\text { Region 3 } \\
\times 10^{-16}\end{array}$ \\
\hline$A$ & 1.4961 & -8.3338 & -1.2126 \\
$B$ & 2.6265 & 3.8947 & 0.0030217 \\
$C$ & -1.2126 & -0.42254 & 0.040622 \\
$D$ & 2.4668 & 1.5988 & -0.0024917 \\
\hline
\end{tabular}

we proposed to perform this transformation was a polynomial algorithm. This algorithm has two stages; the first one transforms the exponential curve into a linear one and once linear, the curve is approximated by using a fourth degree polynomial expressed as

$F(V)=\exp \left(E+D V+C V^{2}+B V^{3}+A V^{4}\right)$
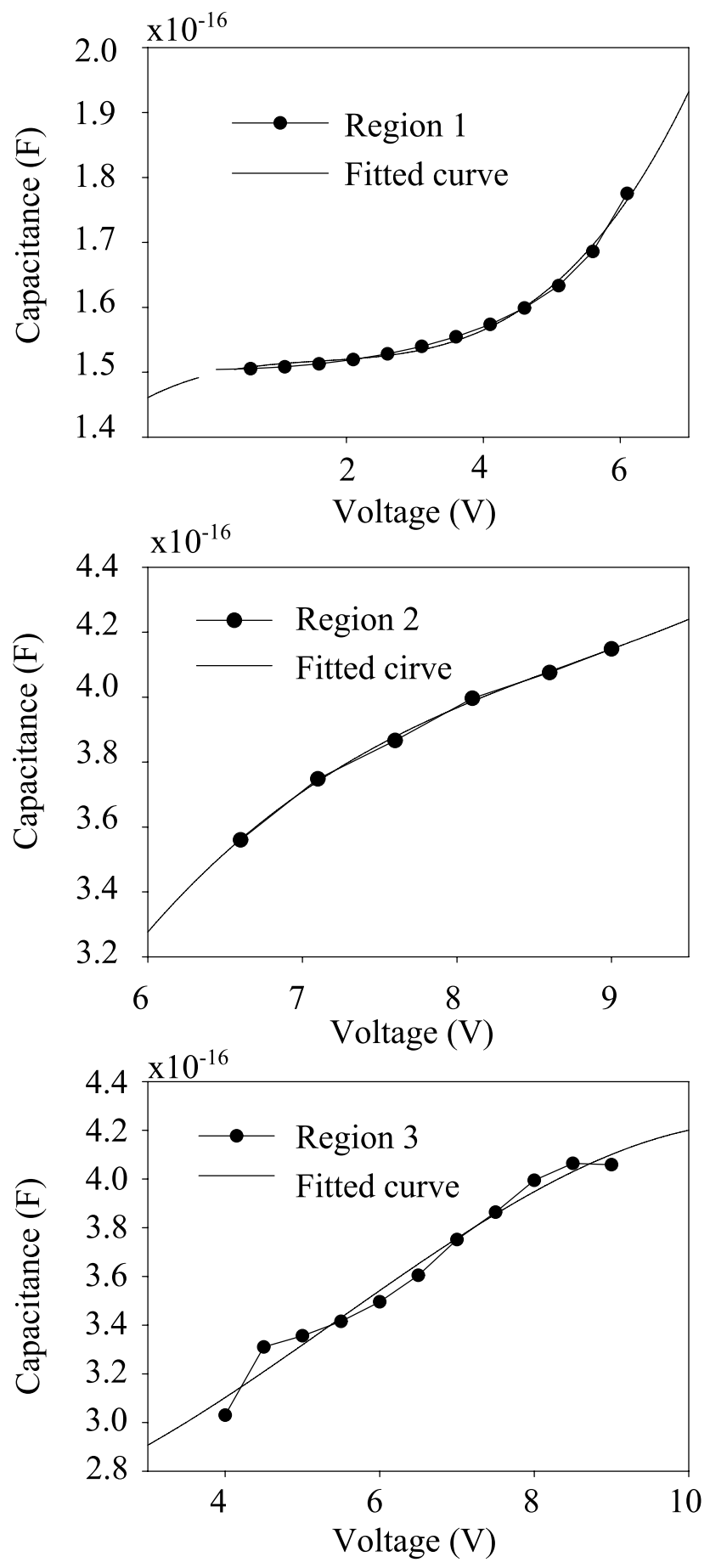

Fig. 19 Regions fitted by a third order algorithm 


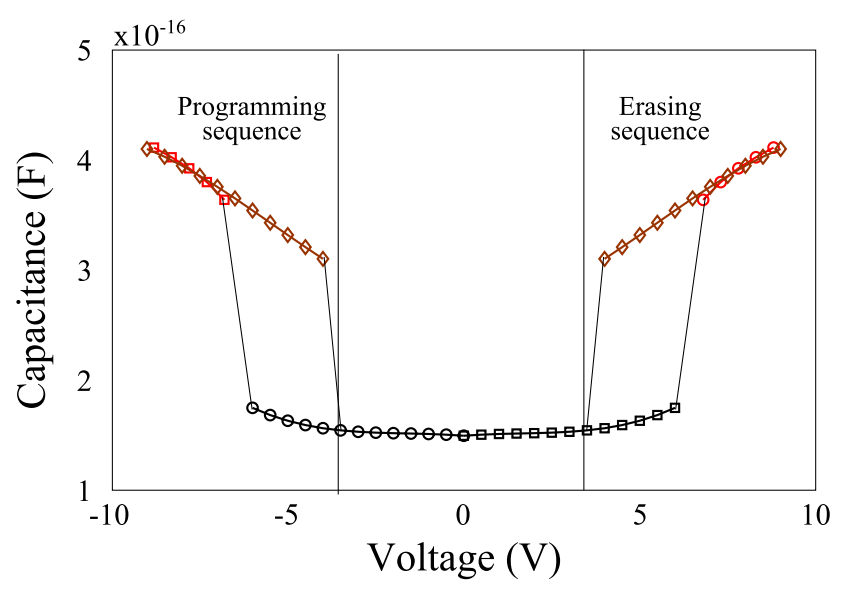

Fig. 20 Pull-in effect curve fitted by a third order polynomial
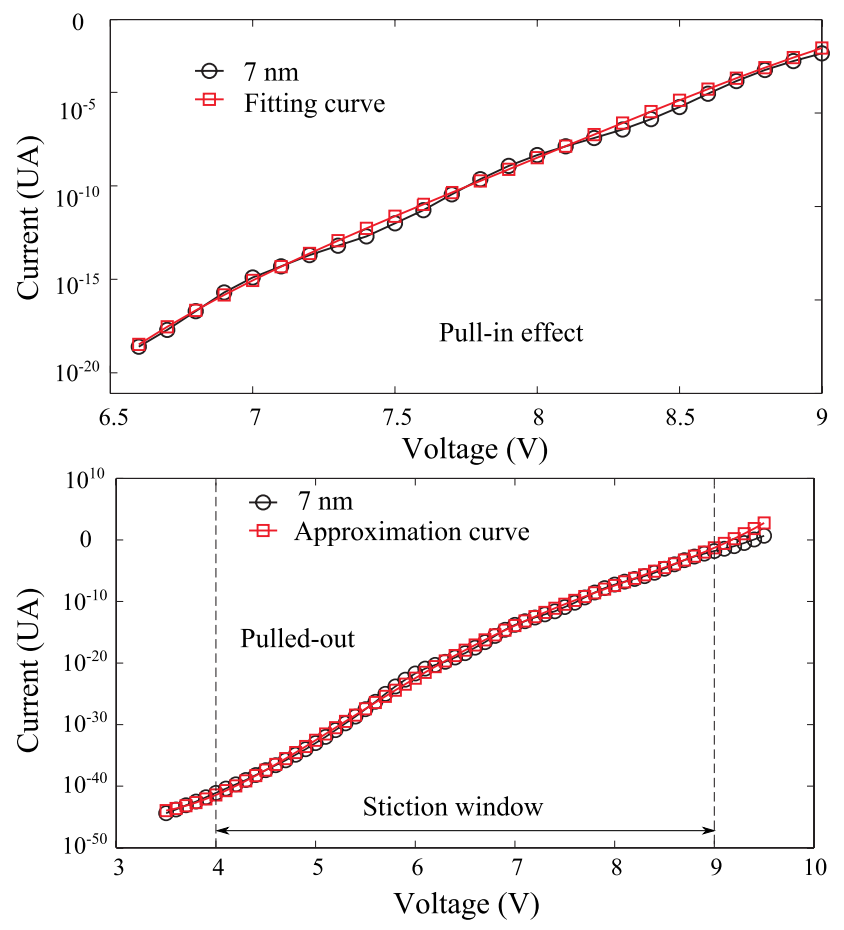

Fig. 21 Current density curve and an approximation by a fourth degree polynomial function

Table 4 Structural parameters for a low-voltage programming sequence

\begin{tabular}{lrrrrr}
\hline \multicolumn{1}{c}{$A$} & \multicolumn{1}{c}{$B$} & $C$ & \multicolumn{1}{l}{$D$} & \multicolumn{1}{l}{$E$} \\
\hline Region 1 & -0.3045 & 9.7618 & -117.4 & 633.95 & \multicolumn{1}{c}{-1316.2} \\
Region 2 & 0.0917 & -2.4718 & 23.829 & -88.631 & 66.547 \\
\hline
\end{tabular}

where the coefficients for each curve are expressed in Table 4.

\section{References}

1. International Technology Roadmap for Semiconductors. ITRS2009 Edition. http://www.itrs.net/Links/2009ITRS/Home2009. htm (2009)

2. Tsu, R., Esaky, L.: Appl. Phys. Lett. 22, 562 (1973)

3. Groeseneken, G., Maes, H., Van Houdt, J., Witters, J.: In: Nonvolatile Semiconductor Memory Technology, pp. 1-88 (1971)

4. Frohman-Bentchkowsky, D.: In: IEDM-81, pp. 14-17 (1981)

5. Tiwari, S., Rana, F., Chan, K., Hanafi, H., Chan, W., Buchanan, D.: In: IEDM, pp. 521-524. IEEE Press, New York (1995)

6. Tiwari, S., Rana, F., Hanafi, H., Hartstein, A., Crabbe, E., Chan, K.: Appl. Phys. Lett. 68, 1377 (1996)

7. Tanaka, A., Tsuchiya, Y., Yamahata, G., Mizuta, H., Oda, S., Usami, K.: Curr. Appl. Phys. 6, 344 (2006)

8. Mizuta, H., Oda, S., Uno, S., Mori, N., Koshida, N.: In: Device Applications of Silicon Nanocrystals and Nanostructures, pp. 197-222. Springer, Berlin (2008)

9. Ionescu, A., Pott, V., Fritschi, R., Banerjee, K., Declercq, M., Renaud, P., Hibert, C., Fluckiger, P., Racine, G.: In: Proceedings International Symposium on Quality Electronic Design, p. 496 (2002)

10. Abelé, N., Fritschi, R., Boucart, K., Casset, F., Ancey, P., Ionescu, A.: In: IEDM, Late News, pp. 479-481 (2005)

11. Nagami, T., Mizuta, H., Momo, N., Tsuchiya, Y., Saito, S., Arai, T., Shimada, T., Oda, S.: IEEE Trans. Electron Devices 54, 1132 (2007)

12. Nagami, T., Tsuchiya, Y., Saito, S., Arai, T., Shimada, T., Mizuta, H., Oda, S.: Jpn. J. Appl. Phys. 48, 114502 (2009)

13. García-Ramírez, M.A., Tsuchiya, Y., Mizuta, H.: In: ESSDERC/CIRC Fringe (ESS-Fringe), Edinburgh (2008)

14. García-Ramírez, M.A., Tsuchiya, Y., Mizuta, H.: Microelectron. Eng. 87, 1284 (2010)

15. Senturia, S.D.: Microsystem Design. Kluwer Academic, Dordrecht (2001)

16. Comsol. Comsol Multiphysics. http://www.comsol.com (2008)

17. CoventorWare. CoventorWare, Designer Reference. http://www. coventor.com/index.html (2008)

18. Rong, H., Huang, Q., Nie, M., Li, W.: Sens. Actuators B, Chem. 116, 15 (2004)

19. Batra, R.C., Porfiri, M., Spinello, D.: Sensors 8, 1048 (2008)

20. Winterton, R.H.S.: Contemp. Phys. 11, 559 (1970)

21. Sze, S.M.: Physics of Semiconductor Devices. Wiley, New York (1981)

22. Using Verilog-A in Advanced Design System (2004)

23. FitzPatrick, D., Miller, I.: Europhys. Lett. (1998)

24. Verilog-A Reference Manual (2003)

25. Abelé, N., et al.: In: International Electron Devices Meeting, IEDM'06 (2006)

26. Nagami, T., et al.: Jpn. J. Appl. Phys. 49, 044304 (2010)

27. Mizuta, H., Tanoue, T.: The Physics and Applications of Resonant Tunnelling Diodes. Cambridge Studies in Semiconductor Physics and Microelectronic Engineering, vol. 2. Cambridge University Press, Cambridge (1994)

28. Tanaka, A., et al.: Jpn. J. Appl. Phys. 47, 3731-3734 (2008)

29. She, M., King, Y.-C., King, T.-J., Hu, C.: In: Device Research Conference, p. 139 (2001)

30. Prince, B.: Emerging Memories: Technologies and Trends. Springer, Berlin (2002)

31. SmartSpice, Manual, Silvaco (2008)

32. Dedkov, G.V., Rekhviashvili, S.S.: Tech. Phys. 44, 982 (1999) 